\title{
Role of clinically determined depth of tumor invasion in the treatment of esophageal carcinoma
}

Thomas W. Rice, MD, ${ }^{a}$ Eugene H. Blackstone, MD, ${ }^{a, b}$ David J. Adelstein, MD, ${ }^{c}$ Gregory Zuccaro, Jr, MD, ${ }^{d}$

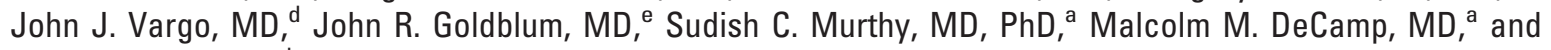
Lisa A. Rybicki, MS

See related editorial on page 988.
From the Center for Swallowing and Esophageal Disorders, Departments of Thoracic and Cardiovascular Surgery, ${ }^{\mathrm{a}}$ Biostatistics and Epidemiology, ${ }^{\mathrm{b}}$ Hematology and Medical Oncology, ${ }^{\mathrm{c}}$ Gastroenterology, ${ }^{\mathrm{d}}$ and Anatomic Pathology, ${ }^{\text {e The Cleveland }}$ Clinic Foundation, Cleveland, Ohio.

Read at the Eighty-first Annual Meeting of The American Association for Thoracic Surgery, San Diego, Calif, May 6-9, 2001.

Received for publication April 12, 2001; revisions requested Aug 7, 2001; revisions received May 30, 2002; accepted for publication June 24, 2002.

Address for reprints: Thomas W. Rice, MD, The Cleveland Clinic Foundation, $9500 \mathrm{Eu}-$ clid Ave/Desk F25, Cleveland, OH 44195 (E-mail: ricet@ccf.org).

J Thorac Cardiovasc Surg 2003;125: 1091-102

Copyright $\odot 2003$ by The American Association for Thoracic Surgery

0022-5223/2003\$30.00+0

doi: $10.1067 / \mathrm{mtc} .2003 .404$
Objective: We sought to evaluate the effectiveness of clinical staging of depth of tumor invasion (cT), the relationship of $\mathrm{cT}$ to survival, the benefits of downstaging $\mathrm{cT}$, and the role of $\mathrm{cT}$ in treatment decisions.

Methods: The accuracy of determining $\mathrm{T}$ by means of endoscopic ultrasonography and the relationship of cT to survival were assessed in 209 patients undergoing esophagectomy alone for esophageal carcinoma. The benefit of downstaging cT was assessed in 128 patients undergoing induction therapy and esophagectomy. The role of $\mathrm{cT}$ in treatment decisions was determined by integrating these results with the results of previous work.

Results: Compared with pathologic T (pT), cT was $87 \%$ accurate, $82 \%$ sensitive, $91 \%$ specific, $89 \%$ positively predictive, and $86 \%$ negatively predictive of tumors confined to $(\leq \mathrm{T} 2)$ or invading beyond $(>\mathrm{T} 2)$ the esophageal wall. In cN0, increasing cT was predictive of progressively poorer survival. For each category of pT N0, cT accurately predicted survival, except for pT3, which was underestimated $(P<$ $.0001)$. In $\mathrm{cN} 0$, downstaging by induction therapy was beneficial only if tumors invaded beyond the wall ( $\geq \mathrm{cT} 3, P=.0003)$. In $\mathrm{cN} 1$, it was beneficial only when downstaging was synchronous in $\mathrm{cT} 3 / \mathrm{T} 4(P<.001)$.

Conclusions: cT should be the principal determinant of treatment in cNO. In cN0, if endoscopic ultrasonography identifies tumors of greater than cT2, multimodality therapy should be considered. However, only when cT3/T4 tumors are downstaged to pT2 or less will patients benefit, but their survival will not equal that of patients with tumors of cT2 or less having esophagectomy alone. If endoscopic ultrasonography identifies tumors of cT2 or less, esophagectomy alone should be used because induction therapy might adversely affect survival.

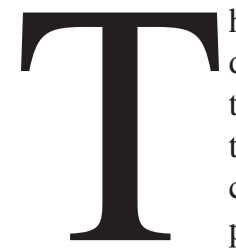

he categorization of TNM staging of esophageal carcinoma reflects disease progression: increasing depth of invasion by the primary tumor $(\mathrm{T})$ to regional lymph node metastases $(\mathrm{N})$ to distant metastases (M). However, in treatment decisions the hierarchy of staging criteria is reversed. Discovery of distant metastases (M1) has dismal prognosis and generally renders patients inoperable ${ }^{1}$; discovery of regional lymph node metastases (N1), with its grave prognosis, makes esophagectomy alone ineffective, leading to a search for successful multimodality therapies. ${ }^{2}$ When esophagectomy was the only therapeutic option, accurate clinical deter- 
TABLE 1. Patient characteristics

\begin{tabular}{|c|c|c|c|c|c|c|c|}
\hline \multirow[b]{3}{*}{ Characteristic } & & & \multicolumn{4}{|c|}{ Treatment } & \multirow[b]{3}{*}{$P$ value } \\
\hline & \multicolumn{2}{|c|}{ Total } & \multicolumn{2}{|c|}{ Esophagectomy alone } & \multicolumn{2}{|c|}{ Induction therapy } & \\
\hline & $\mathbf{n}$ & $\%$ of 337 & n & $\%$ of 209 & n & $\%$ of 128 & \\
\hline \multicolumn{8}{|l|}{ Demography } \\
\hline Male & 280 & 83 & 174 & 83 & 106 & 83 & .9 \\
\hline White & 319 & 95 & 202 & 97 & 117 & 91 & .04 \\
\hline $\begin{array}{l}\text { Age (mean } \pm S D \text { ) } \\
\text { Age range }\end{array}$ & \multicolumn{2}{|c|}{$\begin{array}{l}62 \pm 10 \\
(26-84)\end{array}$} & \multicolumn{2}{|c|}{$\begin{array}{c}63 \pm 11 \\
(33-84)\end{array}$} & \multicolumn{2}{|c|}{$\begin{array}{l}60 \pm 9 \\
(26-77)\end{array}$} & .002 \\
\hline Tumor & & & & & & & .030 \\
\hline Adenocarcinoma & 272 & 81 & 178 & 85 & 94 & 73 & \\
\hline Squamous & 61 & 18 & 29 & 14 & 32 & 25 & \\
\hline Adenosquamous & 4 & 1 & 2 & 1 & 2 & 2 & \\
\hline Differentiation & & & & & & & .01 \\
\hline Poor and moderately poor & 182 & 54 & 101 & 48 & 81 & 63 & \\
\hline Moderate & 85 & 25 & 55 & 26 & 30 & 23 & \\
\hline Well and moderately well & 70 & 21 & 53 & 25 & 17 & 13 & \\
\hline Surgical approach & & & & & & & $<.001$ \\
\hline Thoracotomy & 267 & 79 & 143 & 68 & 124 & 97 & \\
\hline Transhiatal & 69 & 21 & 65 & 31 & 4 & 3 & \\
\hline Laparotomy & 1 & 0.3 & 1 & 0.5 & 0 & 0 & \\
\hline Surgical margins & & & & & & & .2 \\
\hline Positive & 23 & 7 & 17 & 8 & 6 & 5 & \\
\hline No. of N1 nodes & & & & & & & .3 \\
\hline 0 & 181 & 54 & 107 & 51 & 74 & 58 & \\
\hline 1 & 32 & 10 & 18 & 9 & 14 & 11 & \\
\hline 2 & 25 & 7 & 15 & 7 & 10 & 8 & \\
\hline 3 or more & 99 & 29 & 69 & 33 & 30 & 23 & \\
\hline
\end{tabular}

mination of the depth of tumor invasion (cT) was not necessary because the pathologic depth of tumor invasion (pT) provided all the information available for prognostication. Today, the complete spectrum of esophageal carcinoma, from high-grade dysplagia to distant metastatic disease, is seen. Also, many therapeutic options are available. Thus, in patients without distant or regional lymph node metastases, treatment decisions must be made on the basis of $\mathrm{cT}$.

Therefore, the purposes of this study were to evaluate the effectiveness of clinical staging of depth of tumor invasion (cT), the relationship of cT to survival, the benefits of downstaging $\mathrm{cT}$, and the role of $\mathrm{cT}$ in treatment decisions.

\section{Patients and Methods \\ Patients}

Between 1983 and January 1, 2001, 646 patients with esophageal carcinoma underwent esophagectomy at The Cleveland Clinic Foundation. From this database, 337 patients were identified with known clinical (before therapy) and pathologic T, N, and M status (Table 1). ${ }^{3}$

\section{Staging}

Before treatment, clinical depth of tumor invasion (cT) and clinical regional lymph node status $(\mathrm{cN})$ were determined by endoscopic esophageal ultrasonography (EUS) according to the 1997 American Joint Committee on Cancer criteria (Table 2). ${ }^{3}$ EUS definition of $\mathrm{T}$ focused on the fourth ultrasonographic layer to define cT1 to cT3 (Figure 1). cT4 was defined by EUS evidence for invasion of adjacent structures. In these patients EUS and computed tomographic scanning staged all but 7 patients as having $\mathrm{cM} 0$ disease.

After esophagectomy, the pathologic depth of tumor invasion $(\mathrm{pT})$ and pathologic regional lymph node status $(\mathrm{pN})$ were determined (Table 2). The pT categories were p high-grade dysplasia if malignant cells were confined to the epithelium and did not breach the basement membrane, pT1 if the tumor did not invade beyond the submucosa, pT2 if the tumor invaded the muscularis propria but not beyond, pT3 if the tumor invaded the periesophageal tissue, and pT4 if the tumor invaded adjacent structures (Figure 1). ${ }^{3}$ If no residual tumor was found after induction therapy, the patient's status was pT0. At resection, all accessible distant sites were evaluated. Twenty-five patients had pM1a (7\%) tumors, and 10 had pM1b (3\%) tumors.

\section{Treatment}

Of the 337 patients, 209 underwent esophagectomy alone (Table 1). Because this group of patients underwent esophagectomy immediately after clinical staging with no intervening therapies, this provided information about the effectiveness of clinical staging of $\mathrm{T}$ in terms of referent values (accuracy, sensitivity, specificity, positive predictive value, and negative predictive value). It also provided survival data after esophagectomy alone in relation to cT.

The remaining 128 patients received induction therapy after clinical staging and before esophagectomy (Table 1). This group 
TABLE 2. Staging

\begin{tabular}{|c|c|c|c|c|c|c|c|}
\hline \multirow[b]{3}{*}{ Characteristic } & \multirow{2}{*}{\multicolumn{2}{|c|}{ Total }} & \multicolumn{4}{|c|}{ Treatment } & \multirow[b]{3}{*}{$P$ value } \\
\hline & & & \multicolumn{2}{|c|}{ Esophagectomy alone } & \multicolumn{2}{|c|}{ Induction therapy } & \\
\hline & n & $\%$ of 337 & n & $\%$ of 209 & n & $\%$ of 128 & \\
\hline \multicolumn{8}{|l|}{ Clinical stage } \\
\hline cT & & & & & & & $<.001$ \\
\hline HGD & 27 & 8 & 27 & 13 & 0 & 0 & \\
\hline 1 & 24 & 7 & 20 & 10 & 4 & 3 & \\
\hline 2 & 61 & 18 & 43 & 21 & 18 & 14 & \\
\hline 3 & 216 & 64 & 112 & 54 & 104 & 81 & \\
\hline 4 & 9 & 3 & 7 & 3 & 2 & 2 & \\
\hline $\mathrm{cN}$ & & & & & & & $<.001$ \\
\hline 0 & 151 & 45 & 111 & 53 & 40 & 31 & \\
\hline 1 & 186 & 55 & 98 & 47 & 88 & 69 & \\
\hline \multicolumn{8}{|c|}{ Pathologic stage } \\
\hline pT & & & & & & & $<.001$ \\
\hline 0 & 23 & 7 & 0 & 0 & 23 & 18 & \\
\hline HGD & 25 & 7 & 21 & 10 & 4 & 3 & \\
\hline 1 & 80 & 24 & 59 & 28 & 21 & 16 & \\
\hline 2 & 38 & 11 & 17 & 8 & 21 & 16 & \\
\hline 3 & 165 & 49 & 109 & 52 & 56 & 44 & \\
\hline 4 & 6 & 2 & 3 & 1 & 3 & 2 & \\
\hline pN & & & & & & & .2 \\
\hline 0 & 181 & 54 & 107 & 51 & 74 & 58 & \\
\hline 1 & 156 & 46 & 102 & 49 & 54 & 42 & \\
\hline $\mathrm{pM}$ & & & & & & & .17 \\
\hline 0 & 302 & 90 & 189 & 90 & 113 & 88 & \\
\hline $1 \mathrm{a}$ & 25 & 7 & 12 & 6 & 13 & 10 & \\
\hline $1 b$ & 10 & 3 & 8 & 4 & 2 & 2 & \\
\hline
\end{tabular}

$H G D$, High-grade dysplasia.

provided no information about referent values; rather, it provided information relevant to the benefit and role of downstaging $\mathrm{T}$.

Esophagectomy alone. Of the 209 patients undergoing esophagectomy alone, $68 \%$ had resection with 2-field lymphadenectomy by means of thoracotomy. Thirty-one percent had transhiatal esophagectomy with lymph node sampling (Table 1). R0 resections were achieved in $92 \%$.

Induction therapy and esophagectomy. Of the 128 patients receiving induction therapy, 16 patients before 1991 received preoperative chemotherapy only; this included 2 planned courses of cisplatin, etoposide, and doxorubicin. ${ }^{4}$ Since 1991, patients were offered induction chemoradiotherapy for $\mathrm{cN} 1$ and cT3 or cT4 cancers. However, treating physicians had the latitude to treat patients with cT1 N0 $(n=3)$ and cT2 N0 $(n=8)$ cancers with induction therapy. Induction therapy included 2 courses of chemotherapy, either cisplatin and 5-fluorouracil ${ }^{5}$ or cisplatin and taxol. ${ }^{6}$ Concurrent with chemotherapy, accelerated fractionation radiation therapy ( 1.5 Gy twice daily to a total dose of $45 \mathrm{~Gy}$ ) was administered in a split fashion over $4 \frac{1}{2} 2$ weeks. Esophagectomy was performed 4 to 6 weeks later. Ninety-seven percent of patients had esophagectomy with 2-field lymphadenectomy via thoracotomy, and $3 \%$ had transhiatal esophagectomy with lymph node sampling (Table 1). R0 resections were achieved in $95 \%$ of patients.

These treatment protocols had approval and were reviewed annually by The Cleveland Clinic Foundation's Institutional Review Board. Written informed consent was obtained from all patients before treatment began.

\section{Study End Point and Patient Follow-up}

The end point of the study was all-cause mortality. Patients were followed by means of periodic clinic visits and cross-sectional systematic follow-up in February 2001. At that time, 2 patients previously followed for 2.8 and 7.8 years, respectively, could not be located. A search of the Social Security Death Index did not find the Social Security numbers deactivated; it was therefore assumed that the patients were alive 6 months before February 2001 (September 1, 2000). Mean follow-up was $26 \pm 15$ months (43 \pm 35 months in patients alive at last follow-up).

\section{Data Analysis}

Descriptive. Descriptive statistics are summarized as means and SDs for continuous variables and as frequencies and percentages for categorical variables.

Effectiveness of clinical staging of $\boldsymbol{T}$. cT was compared with pT in the group undergoing esophagectomy alone $(n=209)$. For this comparison, clinical and pathologic $\mathrm{T}$ stages were grouped as follows: (1) T2 or less, T2, and greater than T2 and (2) T2 or less and greater than $\mathrm{T} 2$.

Conventional diagnostic characterization is in terms of the dichotomy of "positivity" and "negativity." Because cT is a graded variable, positivity and negativity had to be defined for each tumor depth. In this comparison positivity meant patients had the pT stage being considered, and negativity meant patients did not have the pT stage being considered (either lower or higher $\mathrm{pT}$ ). True positivity meant patients with the $\mathrm{pT}$ stage being considered were 

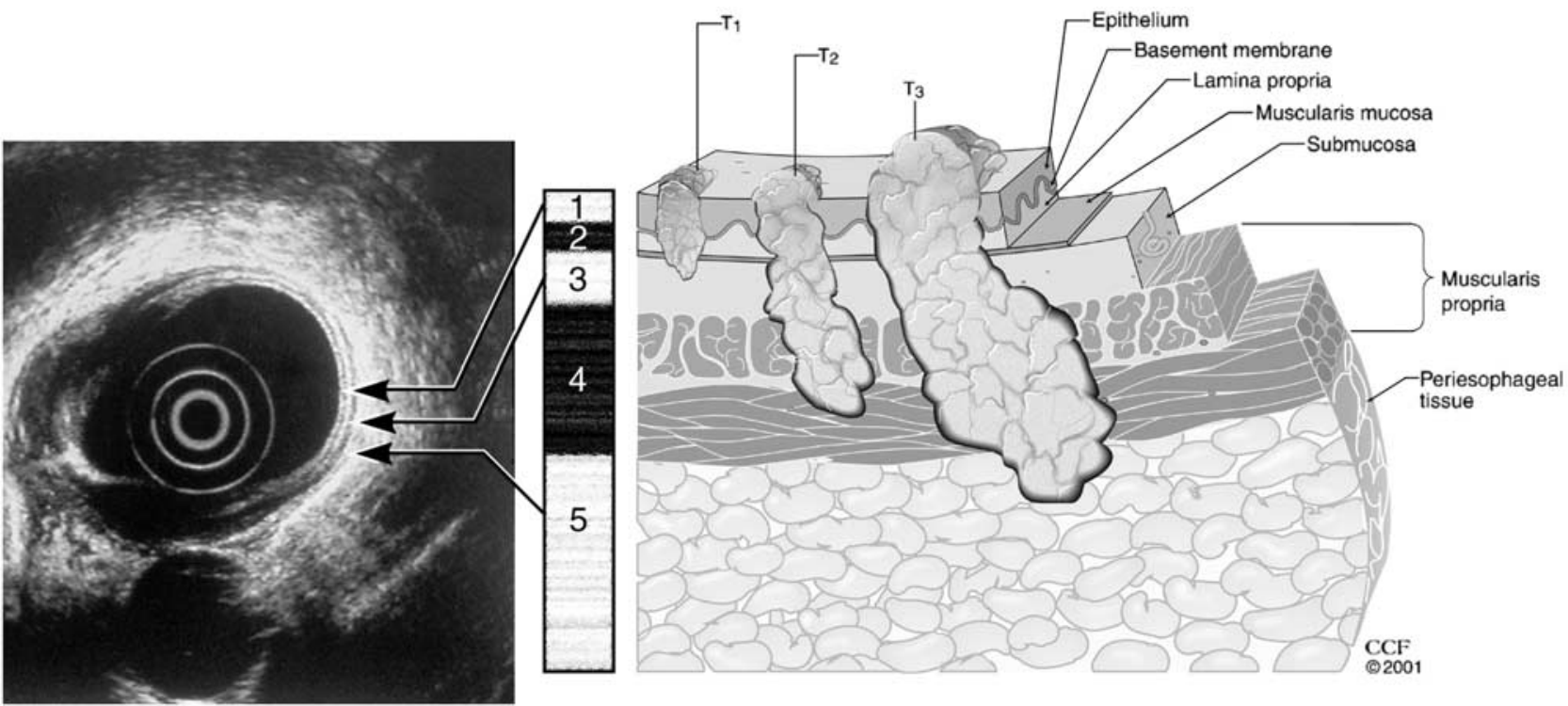

Figure 1. The esophageal wall is visualized as 5 alternating layers of differing echogenicity by means of EUS. The fourth ultrasonographic layer is critical to differentiating T1, T2, and T3 carcinomas. Left and middle, The first (inner) layer is hyperechoic (white) and represents the superficial mucosa (epithelium and lamina propria). The second layer is hypoechoic (black) and represents the deep mucosa (muscularis mucosae). The third layer is hyperechoic and represents the submucosa. The fourth layer is hypoechoic and represents the muscularis propria. The fifth layer is hyperechoic and represents the periesophageal tissue. Right, T1 carcinoma: no invasion beyond the submucosa. The tumor is confined to the first 3 ultrasonographic layers. It does not involve the fourth ultrasonographic layer. T2 carcinoma: invasion into but not beyond the muscularis propria. The tumor is confined to the fourth ultrasonographic layer. T3 carcinoma: invasion into the periesophageal tissue. The tumor breaches the fourth ultrasonographic layer.

correctly clinically staged. False positivity meant patients without the pT stage being considered were incorrectly clinically staged. True negativity meant patients without the pT stage being considered were correctly clinically staged. False negativity meant patients with the pT stage being considered were incorrectly clinically staged. Thus, sensitivity was defined as true-positives divided by the sum of true-positives and false-negatives, specificity as true-negatives divided by the sum of true-negatives and falsepositives, predictive value of a positive test as true-positives divided by the sum of true-positives and false-positives, predictive value of a negative test as true-negatives divided by the sum of true-negatives and false-negatives, and accuracy of staging $\mathrm{T}$ as the sum of true-positives and true-negatives divided by the total number of patients undergoing esophagectomy alone.

Relationship of $\boldsymbol{c T}$ to survival. Prognostication by clinical staging was evaluated by survival analysis of the group undergoing esophagectomy alone $(\mathrm{n}=209)$. Nonparametric estimates of survival were obtained by the Kaplan-Meier method. A parametric method was used to resolve the number of phases of instantaneous risk of death (hazard function) and to estimate their shaping parameters. ${ }^{7}$ Estimates of survival were considered reliable to 10 years. Multivariable analysis of survival in this group was necessary to demonstrate the prognostic value of clinical staging compared with pathologic staging and to provide a risk-adjusted comparison with induction therapy.
For multivariable analyses, only variables known before esophagectomy and the planned surgical approach were examined. Variables included demography (Table 1), depth of tumor invasion by EUS (cT), preoperative assessment of mediastinal nodes $(\mathrm{cN})$, planned surgical approach (Table 1), and date of operation. Depth of tumor invasion was analyzed both as an ordinal variable and as a set of dichotomous variables for each cT level; these produced equivalent models. Two-way interactions of all variables with $\mathrm{cN}$ and surgical approach and 3-way interactions with $\mathrm{cN}$, surgical approach, and the other variables were examined.

Continuous and ordinal variables were assessed univariably by decile risk analysis to suggest transformations of scale required to ensure that relationships of these measured variables were well calibrated to model outcome.

Multivariable analysis was performed simultaneously for 2 hazard phases identified from the data. Both a guided technique of entry of variables into the multivariable models ${ }^{8}$ and bootstrap bagging with 1000 resamplings were used. ${ }^{9}$ A $P$ value of .1 for retention of variables in final models was used.

Patients with pNO M0 tumors undergoing esophagectomy alone were studied to evaluate the relationship of cT to pT in terms of prognostication. For each patient, a survival curve was predicted on the basis of cTN from the multivariable equation. For each category of $\mathrm{pT}$, these survival curves based on cT were averaged and compared with the actual Kaplan-Meier estimates. ${ }^{10}$ To test 
TABLE 3. Accuracy of clinical staging of T for 209 patients undergoing esophagectomy alone

\begin{tabular}{|c|c|c|c|c|c|c|c|c|}
\hline \multirow[b]{2}{*}{ cT } & \multicolumn{3}{|c|}{ pT } & \multirow{2}{*}{$\begin{array}{c}\text { Accuracy } \\
(\%)\end{array}$} & \multirow{2}{*}{$\begin{array}{c}\text { Sensitivity } \\
(\%)\end{array}$} & \multirow{2}{*}{$\begin{array}{c}\text { Specificity } \\
(\%)\end{array}$} & \multirow{2}{*}{$\begin{array}{l}\text { PPV } \\
(\%)\end{array}$} & \multirow{2}{*}{$\begin{array}{l}\text { NPV } \\
(\%)\end{array}$} \\
\hline & $<\mathrm{pT2}$ & pT2 & $>\mathrm{pT} 2$ & & & & & \\
\hline$<\mathrm{cT} 2$ & 46 & 0 & 1 & 83 & 58 & 99 & 98 & 79 \\
\hline cT2 & 24 & 10 & 9 & 81 & 59 & 83 & 23 & 96 \\
\hline$>\mathrm{cT} 2$ & 10 & 7 & 102 & 87 & 91 & 82 & 86 & 89 \\
\hline
\end{tabular}

$N P V$, Negative predictive value; $P P V$, positive predictive value.

for differences between expected and observed survival, cumulative hazard at the time of each patient's follow-up (or death) was calculated from this multivariable equation. The sum of the cumulative hazard values was the number of expected deaths on the basis of cT. A $\chi^{2}$ goodness-of-fit test was used to compare the number of expected and observed deaths. ${ }^{10}$

Benefit of downstaging $\boldsymbol{c T}$. The benefits of downstaging cT by induction therapy were derived from survival analysis. Methods of survival analysis within the induction therapy group $(\mathrm{n}=128)$, including multivariable analysis, mirrored those for the group undergoing esophagectomy alone (see above).

To evaluate the benefit of downstaging cT, we compared actual survival of these patients with predicted survival had they undergone esophagectomy alone. For each patient, a survival curve was predicted on the basis of cTN from the multivariable equation of esophagectomy alone. For selected categories of cT and pT, these survival curves were averaged and compared with the actual Kaplan-Meier estimates and tested for differences between expected and observed survival as described above. ${ }^{10}$

cT and treatment decisions. The benefit of staging $\mathrm{T}$ and the use of induction therapy to downstage $\mathrm{T}$ were integrated with the results of our study on staging and downstaging N1 tumors. ${ }^{2}$ A multivariable analysis of survival in patients undergoing induction therapy was performed to assess the independent benefit of downstaging T. Unlike the analyses above, this analysis included pathologic stage and number and percentage of positive nodes (Tables 1 and 2). In addition, variables were formed for the differences between $\mathrm{cN}$ and $\mathrm{pN}$ and between $\mathrm{cT}$ and $\mathrm{pT}$, their interactions with $\mathrm{cN}$ and $\mathrm{pN}$, and interactions with surgical approach.

\section{Results}

\section{Effectiveness of Clinical Staging of T}

Among 209 patients undergoing esophagectomy alone, clinical staging was $81 \%$ to $87 \%$ accurate, $82 \%$ to $99 \%$ specific, and $79 \%$ to $96 \%$ negatively predictive (Table 3 ). For tumors of T2 or less, EUS had low sensitivity (58\% and 59\%). It also poorly staged $\mathrm{T} 2$ tumors (positive predictive value of only $23 \%$ ). However, in the clinically important distinction of tumors confined to the esophageal wall $(\leq \mathrm{T} 2)$ from those invading beyond $(>\mathrm{T} 2)$, clinical staging by means of EUS was $87 \%$ accurate, $82 \%$ sensitive, $91 \%$ specific, $89 \%$ positively predictive, and $86 \%$ negatively predictive.

\section{Relation of cT to Survival}

Increasing $\mathrm{cT}$ was predictive of progressively poorer survival in patients with $\mathrm{cNO}$ disease after esophagectomy alone by both nonparametric and parametric analyses (Figure 2). In addition to $\mathrm{cT}, \mathrm{cN} 1$, thoracotomy, and older age were risk factors for mortality after esophagectomy alone (Table 4).

For patients with pN0 M0 tumors undergoing esophagectomy alone, the equation (Table 4) based on cT accurately predicted survival $(P>.15$, Figure 3$)$, except for pT3, which was underestimated $(P<.0001)$. In this group 20.6 deaths were predicted, but only 13 were observed. The excess deaths were predicted in the subgroup of patients with pT3 N0 M0 tumors who were understaged (ie, patients in whom N1 nodes had been predicted by EUS [cN1] but who had no regional lymph node metastases at operation).

\section{Benefit of Downstaging cT}

Compared with esophagectomy alone, the benefit of downstaging $\mathrm{cT}$ by induction therapy was evident only in patients with cT3 N0 M0 tumors (Figure 4). In the first 2 years, observed and expected survival was similar; however, after that, patients receiving induction therapy had improved survival. Of the 28 patients with cT3 N0 tumors receiving induction therapy, 12 died. Twenty-five deaths were predicted had the patients undergone esophagectomy alone $(P<.0001)$. This illustrates that in $\mathrm{cN} 0$ tumors invading beyond the esophageal wall, induction therapy provides a survival advantage that is realized after 2 years.

In contrast, harm of induction therapy in patients with cT1 N0 and cT2 N0 tumors is shown in Figure 5. Of 11 patients who underwent induction therapy, 10 died: ie, 7 of cancer, 2 of late treatment toxicity, and 1 of unknown cause. Only 1 patient is alive 91 months after treatment. Two deaths were expected within the range of follow-up had these patients undergone esophagectomy alone $(P<.0001)$.

The less-than-hoped-for benefit of complete sterilization of $\mathrm{cN} 1$ tumors (pT0 N0) is shown in Figure 6. Patients experienced an intermediate level of survival, possibly better than expected $(P=.07)$. Survival was similar for patients with $\mathrm{cN} 0(\mathrm{n}=8)$ and $\mathrm{cN} 1(\mathrm{n}=14)$ tumors who experienced sterilization (pT0 N0, $P=.5$ ). Of 22 patients, 10 died: ie, 4 of cancer, 4 of treatment toxicity, and 2 of causes unrelated to cancer. 


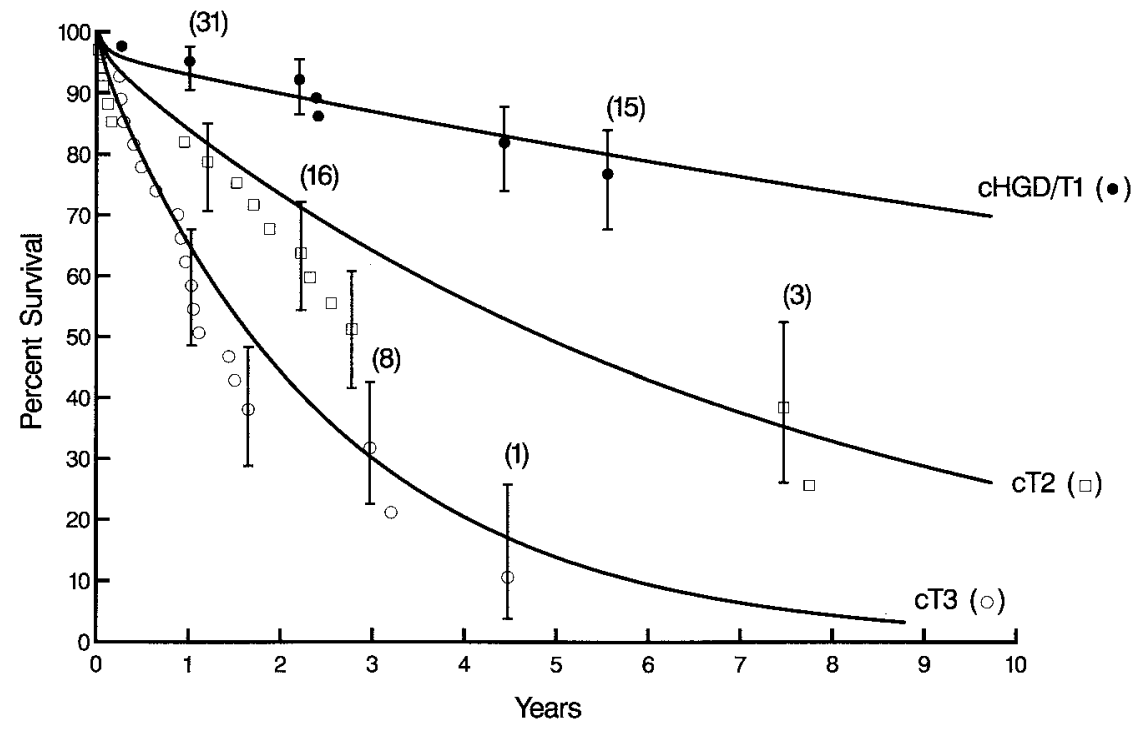

Figure 2. Survival after esophagectomy alone for cNO esophageal cancer stratified according to cT. Symbols represent Kaplan-Meier estimates for each clinically determined depth of tumor invasion and are accompanied by vertical bars representing asymmetric $68 \%$ confidence limits of these estimates (equivalent to $\pm 1 \mathrm{SE}$ ). The numbers in parentheses represent survivors. The smooth curves are parametric estimates of survival for each clinical stage. HGD, High-grade dysplasia.

TABLE 4. Risk factors for mortality after esophagectomy for patients undergoing esophagectomy alone $(\mathrm{n}=\mathbf{2 0 9})$

\begin{tabular}{|c|c|c|c|c|}
\hline \multirow[b]{3}{*}{ Incremental risk factor } & \multicolumn{4}{|c|}{ Hazard phase } \\
\hline & \multicolumn{2}{|c|}{ Early } & \multicolumn{2}{|c|}{ Late } \\
\hline & Coefficient \pm SD & $P$ value & Coefficient \pm SD & $P$ value \\
\hline \multicolumn{5}{|l|}{ Demography } \\
\hline Older age & - & - & $0.62 \pm 0.189$ & .001 \\
\hline \multicolumn{5}{|l|}{ Tumor characteristics } \\
\hline cN1 & 10.7 & $<.001$ & $1.65 \pm 0.63$ & .01 \\
\hline Increasing cT & - & - & $0.92 \pm 0.20$ & $<.0001$ \\
\hline cT4 & $1.54 \pm 0.59$ & .009 & - & - \\
\hline \multicolumn{5}{|l|}{ Planned surgery } \\
\hline Thoracotomy & - & - & $1.24 \pm 0.40$ & .002 \\
\hline
\end{tabular}

\section{cT and Treatment Decisions}

Risk factors for death after induction therapy included older age, female, increasing number of positive nodes, and less downstaging of $\mathrm{cT}$ (Table 5). Figure 7 shows that there must be synchronous downstaging of both $\mathrm{cT}$ and $\mathrm{cN}$ to improve survival (Appendix 1). Patients with $\mathrm{cN} 1$ tumors who downstaged to pNO but still had tumor invasion beyond the esophageal wall ( $\geq$ pT3) had poor survival despite induction therapy and esophagectomy. If there is synchronous downstaging of $\mathrm{cT}$ and $\mathrm{cN}$, there is a homogenization of survival (Figure 7).

\section{Discussion}

\section{Context}

Tumor progression, and therefore TNM staging, is focused on primary tumor $(\mathrm{T})$, then regional nodes $(\mathrm{N})$, and, finally, distant sites (M). Prognosis is determined in reverse order of this progression. Because $\mathrm{T}$ is the easiest to assess noninvasively, it has become the focus of attention in clinical staging. The impetus for this study was to determine when clinical depth of tumor invasion (cT) was of importance in making decisions in the treatment of esophageal carcinoma.

When palliation with single-modality therapy was the aim of treatment, there was no need for clinical staging. Clinical staging has become important since the introduction and increasing use of multimodality therapy. The effect of treatment decisions in neoadjuvant therapy, made on the basis of clinical staging, is difficult to assess because therapy might downstage tumors or there might be disease progression during therapy. In addition, the ability to clinically stage the tumor after neoadjuvant therapy might be 


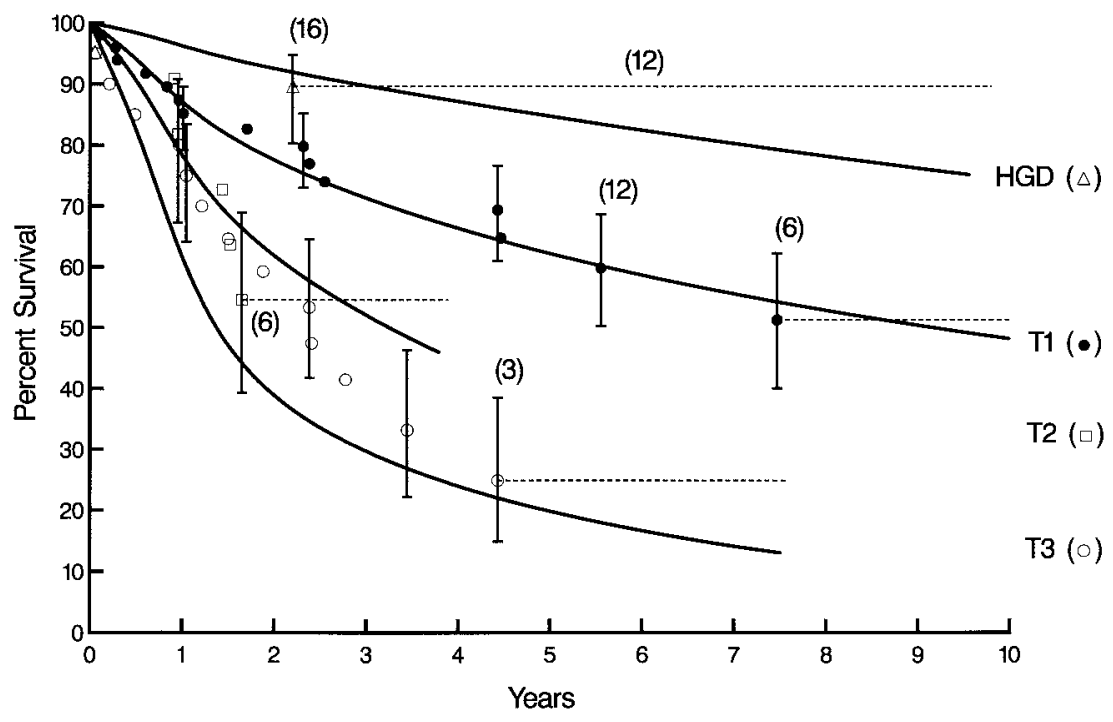

Figure 3. Survival after esophagectomy alone for pNO MO esophageal cancer stratified on the basis of pT. Symbols represent Kaplan-Meier estimates for each pathologic depth of tumor invasion (pT). Fine dashed lines represent survivors beyond the last recorded death. Solid curves represent survival for each pathologic depth of tumor invasion predicted from the multivariable analysis (Table 4) based on clinical depth of tumor invasion (cT). HGD, High-grade dysplasia.

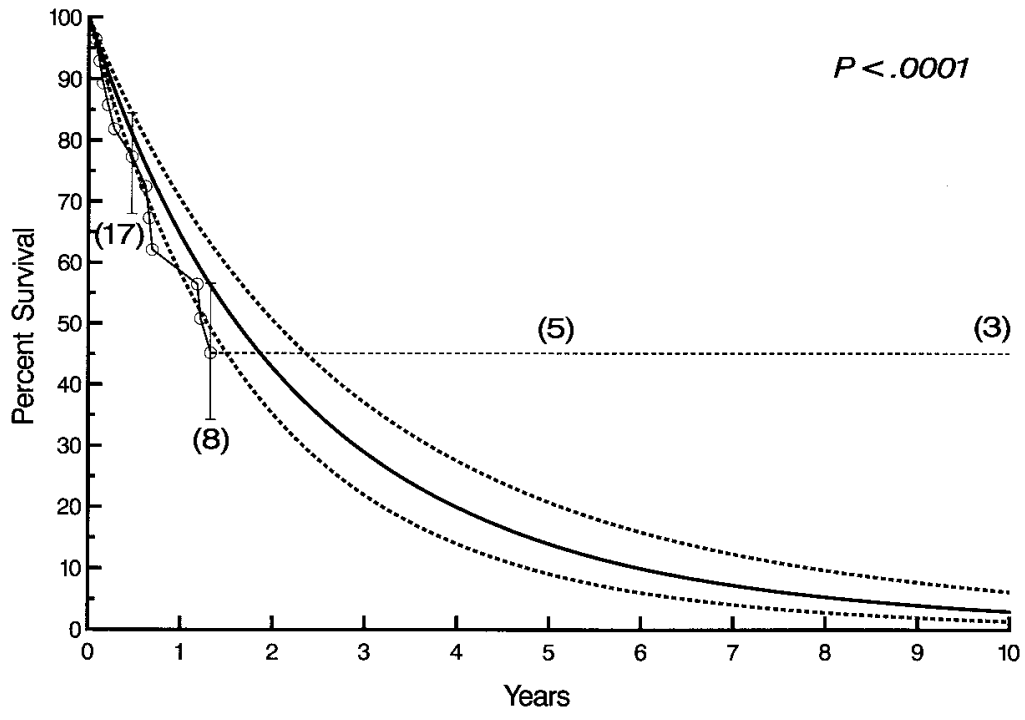

Figure 4. Observed and predicted survival in patients with cT3 NO tumors receiving induction therapy. Open circles represent Kaplan-Meier estimates of observed survival. Vertical bars represent asymmetric $68 \%$ confidence limits of these estimates (equivalent to $1 \mathrm{SE}$ ). Numbers in parentheses represent number of patients traced beyond that point. The dashed line represents survivors beyond the last death recorded. The smooth curve enclosed by its confidence limits is predicted survival had patients undergone esophagectomy alone (Table 4).

lost. ${ }^{11}$ Therefore, it is crucial to have effective and accurate clinical staging and a clear relationship of staging to prognosis to assess the benefit of current treatment and to direct future treatment decisions.

\section{Effectiveness of Clinical Staging of T}

There is a misconception that accuracy of cT determination by EUS increases incrementally with deeper tumor penetration. ${ }^{12}$ In a meta-analysis the reported accuracy was $84 \%$ for 


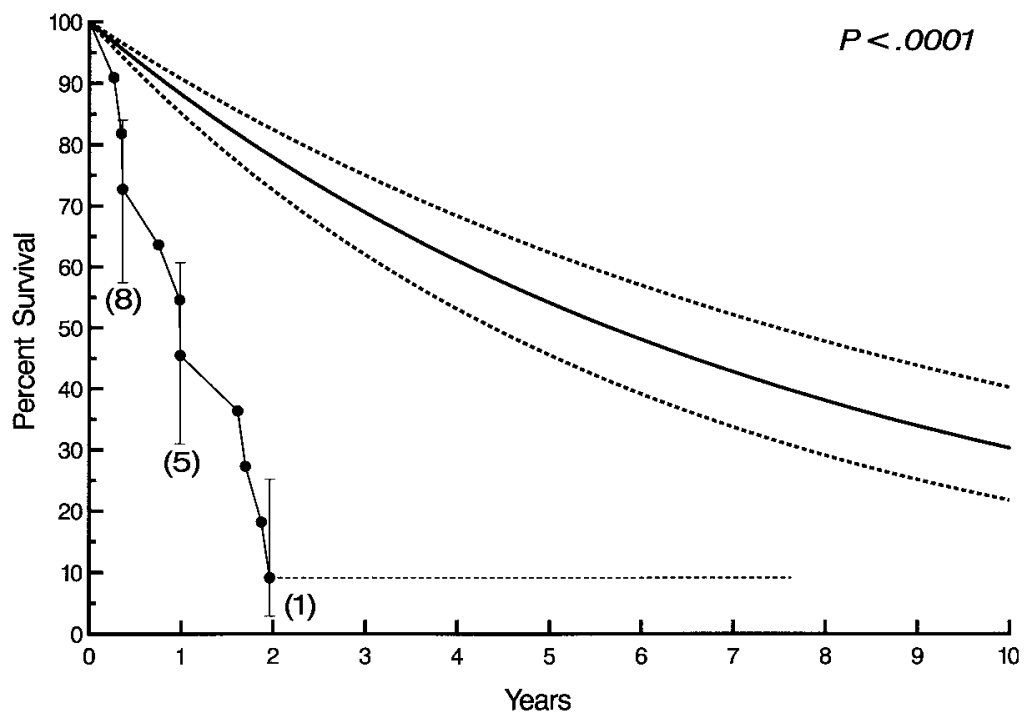

Figure 5. Observed and predicted survival in patients with cT1 NO and cT2 NO tumors receiving induction therapy. The format is the same as for Figure 2, except Kaplan-Meier estimates are shown as filled circles.

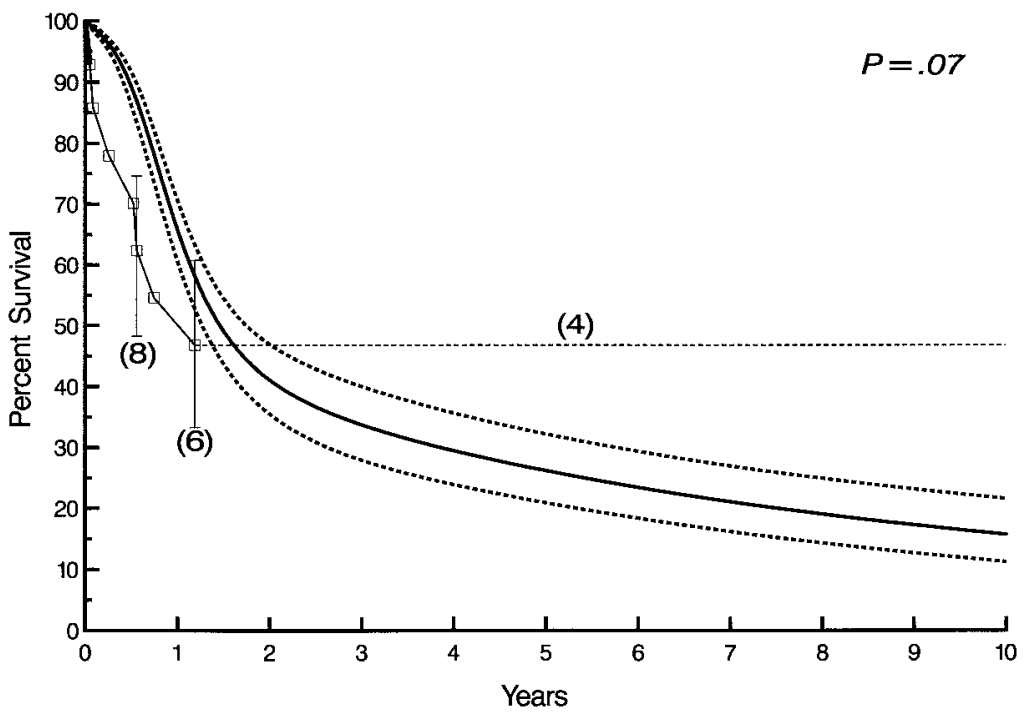

Figure 6. Observed and predicted survival in patients with $\mathrm{cN} 1$ tumors with any $\mathrm{cT}$ receiving induction therapy who were found at operation to have a sterilized tumor (pTO NO). The format is similar to that of Figure 4.

$\mathrm{T} 1,73 \%$ for $\mathrm{T} 2,89 \%$ for $\mathrm{T} 3$, and $89 \%$ for T4 tumors. ${ }^{13} \mathrm{~A}$ review of the literature shows variation in accuracy with $\mathrm{T}$ : $75 \%$ to $82 \%$ for $\mathrm{T} 1,64 \%$ to $85 \%$ for $\mathrm{T} 2,89 \%$ to $94 \%$ for T3, and $88 \%$ to $100 \%$ for T4 tumors. ${ }^{14}$ The least reliable of EUS cT determinations is cT2. ${ }^{15}$ Except for T2 tumors, EUS accuracy increases with increasing $\mathrm{T}$.

In this study the determination of $\mathrm{T}$ by EUS was accurate, specific, and negatively predictive. For tumors invading beyond the esophageal wall $(>\mathrm{T} 2)$, EUS was also sensitive and positively predictive. However, EUS sensitivity in screening for less than T2 and T2 esophageal tumors was poor because of high false negativity. The positive predictive value of EUS determination of T2 was also poor because of high false positivity.

The interplay of staging criteria and EUS anatomy partially accounts for the problem with T2. The muscularis propria is critical in differentiating $\mathrm{T} 1, \mathrm{~T} 2$, and T3 tumors. Tumors are defined as T1 if there is no invasion of the muscularis propria, T2 if invasion is into the muscularis propria, and T3 if invasion is beyond the muscularis propria. For clinical evaluation, the fourth ultrasonographic layer is interpreted as the muscularis propria. However, studies have 


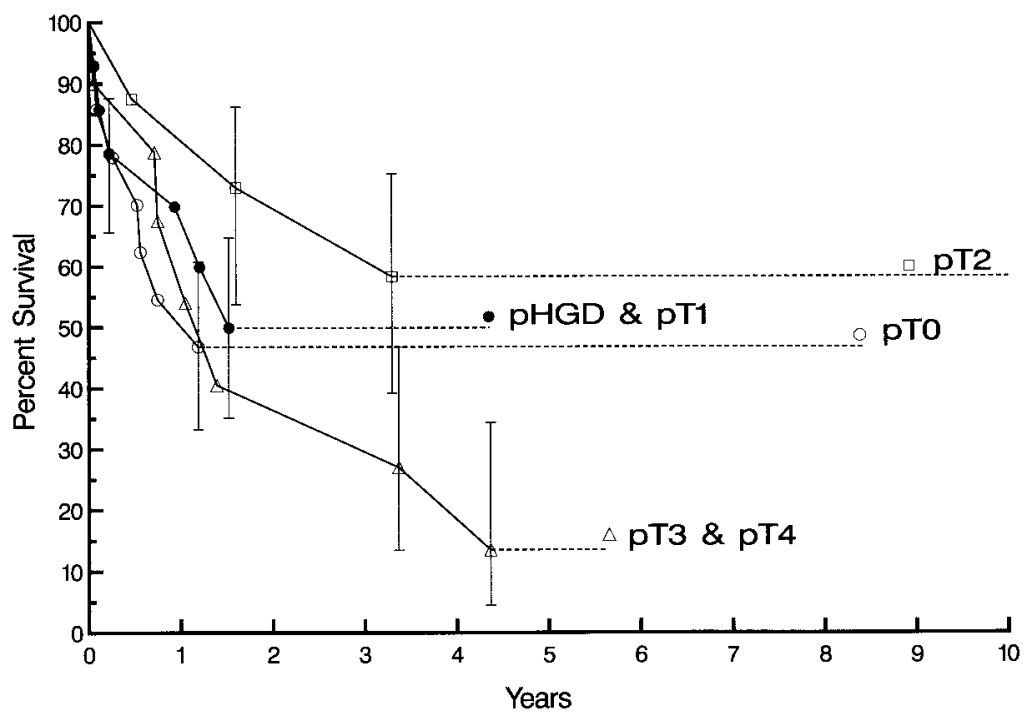

Figure 7. Kaplan-Meier estimates of survival in patients with $\mathrm{cN} 1$ tumors after induction therapy resulting in pNO disease, according to residual pathologic depth of tumor invasion (pT). Fifteen cN1 patients had pTO disease, 2 had p high-grade dysplasia (pHGD) and 12 pT1 disease, 8 had pT2 disease, 9 had pT3 disease, and 1 had pT4 disease. Kaplan-Meier estimates are depicted as in Figure 4.

TABLE 5. Incremental risk factors for mortality after esophagectomy in patients who received induction therapy $(\mathbf{n}=128)$

\begin{tabular}{|c|c|c|c|c|}
\hline \multirow[b]{3}{*}{ Incremental risk factor } & \multicolumn{4}{|c|}{ Hazard phase } \\
\hline & \multicolumn{2}{|c|}{ Early } & \multicolumn{2}{|c|}{ Late } \\
\hline & Coefficient \pm SD & $P$ value & Coefficient \pm SD & $P$ value \\
\hline \multicolumn{5}{|l|}{ Demography } \\
\hline Older age* & $-2.8 \pm 1.12$ & .02 & - & - \\
\hline Female & - & - & $1.46 \pm 0.69$ & .03 \\
\hline \multicolumn{5}{|l|}{ Tumor characteristics } \\
\hline No. of positive nodest & - & - & $-5.3 \pm 2.0$ & .009 \\
\hline Change in T if patient had pNO disease $\ddagger$ & - & - & $4.1 \pm 1.64$ & .01 \\
\hline
\end{tabular}

*[50/age] inverse transformation.

$\dagger[1 /$ (number of positive nodes +1$)]$ inverse transformation.

$\ddagger$ See "Patients and Methods" section for expression.

demonstrated that the third ultrasonographic layer is the submucosa and the acoustic interface between the submucosa and muscularis propria. The fourth ultrasonographic layer is the muscularis propria minus the acoustic interface between the submucosa and the muscularis propria. ${ }^{16,17}$ Therefore, the boundary necessary to completely differentiate $\mathrm{T} 2$ from $\mathrm{T} 3$ tumors is contained in the third ultrasonographic layer. Because 2 boundaries must be evaluated in the determination of $\mathrm{T} 2$ and errors might occur at each, the inaccuracy is potentially double that of T1 and T3 tumors.

However, for the clinically pertinent distinction of tumors confined to the esophageal wall $(\leq \mathrm{T} 2)$ from those invading beyond ( $>\mathrm{T} 2)$ the esophageal wall, EUS is highly accurate, specific, sensitive, positively predictive, and negatively predictive.

\section{Relationship of cT to Survival}

Clinical staging of $\mathrm{T}$ is prognostic. Clinical staging of tumors confined to the esophageal wall $(<\mathrm{cT} 2)$ and pathologic staging of these same tumors are similarly predictive of prognosis. Clinical staging of tumors invading beyond the esophageal wall (>cT2) underestimates survival compared with pathologic staging. The interplay of $\mathrm{T}$ and $\mathrm{N}$ in these locally advanced tumors accounts for this underestimation of survival. The majority of patients $(\geq 80 \%)$ with tumors invading beyond the esophageal wall $(>\mathrm{T} 2)$ will have regional lymph node metastases. ${ }^{18}$ However, in the minority without regional lymph node metastases (pNO), clinical overstaging of regional lymph node status $(\mathrm{cN} 1)$, not inaccuracy in predicting depth of tumor invasion $(>\mathrm{cT} 2)$ accounts for the underestimation of survival. 


\section{Benefit of Downstaging cT}

For patients with cNO tumors, survival was enhanced by induction therapy only for those with tumors invading beyond the wall $(>\mathrm{cT} 2)$ who were downstaged. Induction therapy might reduce survival for patients with cT2 N0 or less tumors. For patients with $\mathrm{cN} 1$ tumors downstaged to pNO by induction therapy, survival was enhanced compared with that in patients not receiving induction therapy or those not downstaged by induction therapy. ${ }^{2}$ For tumorss invading beyond the wall ( $>\mathrm{cT} 2$ ), if there was downstaging of $\mathrm{N}$ but not $\mathrm{T}$ or vice versa, the survival advantage of induction therapy was lost.

\section{cT and Treatment Decisions}

Treatment decisions should be based on cT only in patients with cN0 M0 tumors. Patients with esophageal carcinomas that have not metastasized to regional lymph nodes $(\mathrm{cN} 0)$ or breached the esophageal wall $(\leq \mathrm{cT} 2)$ have good survival when treated with esophagectomy alone. Every effort should be made to stage patients clinically and to identify those with esophageal carcinomas confined to the esophageal wall $(\leq \mathrm{cT} 2 \mathrm{~N} 0 \mathrm{M} 0)$ because they are the best surgical candidates and might be harmed by aggressive multimodality therapy. Once tumors have spread beyond the esophageal wall $(>\mathrm{cT} 2)$ or metastasized to regional $(\mathrm{cN} 1)$ or nonregional (cM1a) lymph nodes, survival is drastically reduced. Only patients with advanced tumors whose disease is downstaged to pT2 N0 or less with induction therapy will benefit from this therapy.

\section{Limitations}

This study is a small, nonrandomized, single-institution, prospective experience. There might have been inaccuracies of clinical staging and treatment biases over time. Analysis was made difficult by selection of induction therapy for individual patients. Therefore, inferences from application of information on surgical intervention alone to the induction therapy group are not as robust as they would be had this been a randomized trial. In addition, patients in whom induction therapy was used almost universally received a thoracotomy, and this factor in itself adds risk to the treatment program, ${ }^{19}$ only one component of which is induction therapy.

We thank Diane Baisden for data collection and follow-up and Mary Ann Meljac and Tess Knerik for manuscript preparation.

\section{References}

1. Christie NA, Rice TW, Goldblum JR, Adelstein DJ, Rybicki LA, Blackstone EH. M1a/M1b esophageal carcinoma: surgical relevance. J Thorac Cardiovasc Surg. 1999;118:900-7.

2. Rice TW, Blackstone EH, Adelstein DJ, Zuccaro G Jr, Vargo JJ, Goldblum JR, et al. N1 esophageal carcinoma: the importance of staging and downstaging. J Thorac Cardiovasc Surg. 2001;121:45464.
3. American Joint Committee on Cancer. AJCC cancer staging manual. 5th ed. Philadelphia: Lippincott-Raven; 1997. p. 65-8.

4. Rice TW, Boyce GA, Sivak MV Jr, Adelstein DJ, Kirby TJ. Esophageal carcinoma: esophageal ultrasound assessment of preoperative chemotherapy. Ann Thorac Surg. 1992;53:972-7.

5. Adelstein DJ, Rice TW, Becker M, Larto MA, Kirby TJ, Koka A, et al. Use of concurrent chemotherapy, accelerated fractionation radiation, and surgery for patients with esophageal carcinoma. Cancer. 1997;80:1011-20.

6. Adelstein DJ, Rice TW, Rybicki LA, Larto MA, Ciezki J, Saxton J, et al. Does paclitaxel improve the chemoradiotherapy of locoregionally advanced esophageal cancer? A nonrandomized comparison with fluorouracil-based therapy. J Clin Oncol. 2000;18:2032-9.

7. Blackstone EH, Naftel DC, Turner ME Jr. The decomposition of time-varying hazard into phases, each incorporating a separate stream of concomitant information. J Am Stat Assoc. 1986;81:615-24.

8. Baskerville JC, Toogood JH. Guided regression modeling for prediction and exploration of structure with many explanatory variables. Technometrics. 1982;24:9-17.

9. Breiman L. Bagging predictors. Machine Learning. 1996;24:123-40.

10. Ferrazzi P, McGiffin DC, Kirklin JW, Blackstone EH, Bourge RC. Have the results of mitral valve replacement improved? J Thorac Cardiovasc Surg. 1986;92:186-97.

11. Zuccaro G Jr, Rice TW, Goldblum JR, Mendendorp SV, Becker ME, Pimentel R, et al. Endoscopic ultrasound cannot determine suitability for esophagectomy after aggressive chemoradiotherapy for esophageal cancer. Am J Gastroenterol. 1999;94:906-12.

12. Buenaventure P, Luketich JD. Surgical staging of esophageal cancer. Chest Surg Clin North Am. 2000;10:487-97.

13. Rösch T. Endosonographic staging of esophageal cancer: a review of literature results. Gastrointest Endosc Clin N Am. 1995;5:537-47.

14. Saunders HS, Wolfman NT, Ott DJ. Esophageal cancer. Radiologic staging. Radiol Clin North Am. 1997;35:281-94.

15. Heidemann J, Schilling MK, Schmassmann A, Maurer CA, Buchler MW. Accuracy of endoscopic ultrasonography in preoperative staging of esophageal carcinoma. Dig Surg. 2000;17:219-24.

16. Kimmey MB, Martin RW, Haggitt RC, Wang KY, Franklin DW, Silverstein FE. Histologic correlates of gastrointestinal ultrasound images. Gastroenterology. 1989;96:433-41.

17. Bolondi L, Casenova P, Santi V, Caletti G, Barbara L, Labo G. The sonographic appearance of the normal gastric wall: an in vivo study. Ultrasound Med Biol. 1986;12:991-8.

18. Rice TW, Zuccaro G Jr, Adelstein DJ, Rybicki LA, Blackstone EH, Goldblum JR. Esophageal carcinoma: depth of tumor invasion is predictive of regional lymph node status. Ann Thorac Surg. 1998;65: 787-92.

19. Rice TW, Blackstone EH, Goldblum JR, DeCamp MM, Murthy SC, Falk GW, et al. Superficial adenocarcinoma of the esophagus. J Thorac Cardiovasc Surg. 2001;122:1077-90.

20. Hosmer DW Jr, Lemeshow S. Applied logistic regression. New York: John Wiley \& Sons; 1989. p. 216-38.

\section{Appendix 1}

\section{Linkage of $\mathbf{T}$ and $\mathbf{N}$ After Induction Therapy}

The linkage between $\mathrm{T}$ and $\mathrm{N}$ after induction therapy $(\mathrm{n}=126)$ was assessed by comparing $\mathrm{cT}$ and $\mathrm{cN}$ with $\mathrm{pT}$ and $\mathrm{pN}$.

\section{Method of Assessing Linkage}

High-grade dysplasia and cT1 groups were coalesced to facilitate the analyses. Clinical stage was then numerically scored: $1=\mathrm{cT} 1$ or less; $2=\mathrm{cT} 2 ; 3=\mathrm{cT} 3$; and $4=\mathrm{cT} 4$. A similar score was assigned to pT, except that absence of disease (pT0) was scored as zero. Change in $\mathrm{T}$ was obtained by subtracting the $\mathrm{cT}$ score from the $\mathrm{pT}$ score. Therefore, a negative difference indicated $\mathrm{pT}$ was less than $\mathrm{cT}$ (downstaging or overstaging), and a positive difference indicated $\mathrm{pT}$ was greater than $\mathrm{cT}$ (disease progression or understaging). 


\section{Appendix TABLE 1. Change in T for 128 patients undergoing induction therapy}

\begin{tabular}{|c|c|c|c|c|c|c|}
\hline Variable & n & Mean \pm SD & Median & Minimum & Maximum & $P$ value \\
\hline CT & & & & & & .002 \\
\hline$\leq \mathrm{cT} 1$ & 4 & $0.5 \pm 0.6$ & 0.5 & 0 & 1 & \\
\hline cT2 & 18 & $-0.2 \pm 1.1$ & 0 & -2 & 2 & \\
\hline cT3 & 104 & $-1.0 \pm 1.2$ & 0 & -3 & 1 & \\
\hline cT4 & 2 & $-2.5 \pm 0.7$ & -2.5 & -3 & -2 & \\
\hline pT & & & & & & $<.001$ \\
\hline pTO & 23 & $-2.9 \pm 0.3$ & -3 & -3 & -2 & \\
\hline $\mathrm{pHGD/pT1}$ & 25 & $-1.7 \pm 0.7$ & -2 & -3 & 0 & \\
\hline pT2 & 21 & $-0.6 \pm 0.7$ & -1 & -2 & 1 & \\
\hline pT3 & 56 & $0.1 \pm 0.3$ & 0 & 0 & 1 & \\
\hline pT4 & 3 & $1.3 \pm 0.6$ & 1 & 1 & 2 & \\
\hline $\mathrm{cN}$ & & & & & & 1 \\
\hline $\mathrm{cNO}$ & 40 & $-0.9 \pm 1.4$ & 0 & -3 & 2 & \\
\hline $\mathrm{cN} 1$ & 88 & $-0.9 \pm 1.2$ & 0 & -3 & 1 & \\
\hline $\mathrm{pN}$ & & & & & & $<.001$ \\
\hline pNO & 74 & $-1.4 \pm 1.3$ & -2 & -3 & 2 & \\
\hline pN1 & 54 & $-0.1 \pm 0.7$ & 0 & -3 & 1 & \\
\hline Change in $\mathrm{N}$ ( $\mathrm{cN}$ to $\mathrm{pN}$ ) & & & & & & $<.001$ \\
\hline cN1 to pNO & 46 & $-1.6 \pm 1.2$ & -2 & -3 & 1 & \\
\hline cNO to pNO & 28 & $-1.2 \pm 1.5$ & -1 & -3 & 2 & \\
\hline cN1 to pN1 & 12 & $-0.1 \pm 0.5$ & 0 & -1 & 1 & \\
\hline cN0 to pN1 & 42 & $-0.1 \pm 0.7$ & 0 & -3 & 1 & \\
\hline pM & & & & & & .06 \\
\hline pM0 & 113 & $-1.0 \pm 1.3$ & 0 & -3 & 2 & \\
\hline pM1 & 15 & $-0.3 \pm 0.6$ & 0 & -2 & 0 & \\
\hline Cell type & & & & & & .002 \\
\hline $\mathrm{AD} / \mathrm{AS}$ & 96 & $-0.7 \pm 1.2$ & 0 & -3 & 2 & \\
\hline SO & 32 & $-1.5 \pm 1.3$ & -2 & -3 & 1 & \\
\hline Differentiation & & & & & & .03 \\
\hline Poor or moderately poor & 81 & $-0.7 \pm 1.2$ & 0 & -3 & 2 & \\
\hline Moderately & 30 & $-1.3 \pm 1.3$ & -1.5 & -3 & 1 & \\
\hline Moderately well or well & 17 & $-1.2 \pm 1.3$ & -1 & -3 & 1 & \\
\hline
\end{tabular}

$H G D$, High-grade dysplasia; $A D / A S$, adenocarcinoma and adenosquamous cell carcinoma; S0, squamous cell carcinoma.

Change in $\mathrm{T}$ was analyzed both univariably and multivariably with respect to sex, race, $\mathrm{cT}, \mathrm{pT}, \mathrm{cN}, \mathrm{pN}, \mathrm{pM}$, cell type, tumor differentiation, and surgical margin. Either the Wilcoxon rank sum test (dichotomous variables) or the Kruskal-Wallis test (variables with $>2$ categories) was used to assess the association of these variables to change in $\mathrm{T}$.

For multivariable analysis, change in $\mathrm{T}$ was analyzed as an ordinal variable with 5 levels $(-3,-2,-1,0$, and $\geq 1) .{ }^{20}$ Particular attention was paid to 4 possible combinations of $\mathrm{N}$ : $\mathrm{cN} 0 / \mathrm{pN} 0$, $\mathrm{cN} 1 / \mathrm{pN} 0, \mathrm{cN} 1 / \mathrm{pN} 1$, and $\mathrm{cN} 0 / \mathrm{pN} 1$. The assumption of proportional odds was reasonable, and therefore multivariable ordinal logistic regression was used, which produces a different intercept for each level of change of T. Except for combinations of N, variables were retained in the analysis whose $P$ value was greater than .1 .

\section{Linkage of $\mathbf{T}$ and $\mathbf{N}$}

cT was greater than pT in $46 \%$ of patients (downstaged or overstaged), cT was equal to $\mathrm{pT}$ in $47 \%$, and cT was less than $\mathrm{pT}$ in $7 \%$ (disease progression or understaged). The higher $\mathrm{cT}$, the lower $\mathrm{pT}$ was after induction therapy (pT-cT more negative, Appendix Table 1). The higher $\mathrm{pT}$, the more likely the tumor had remained unchanged or progressed (pT-cT more positive). The difference between cT and pT was similar irrespective of whether there was $\mathrm{cN1}(P=1)$. However, if the patient had pN0 tumors after induction therapy, the magnitude of $\mathrm{T}$ decrease was greater $(-1.4$ for $\mathrm{pN} 0$ vs -0.1 for $\mathrm{pN} 1)$. The magnitude of $\mathrm{T}$ decrease was greatest in patients with pN0 tumors who had been downstaged from $\mathrm{cN} 1$ (ie, downstaging of $\mathrm{T}$ and $\mathrm{N}$ were synchronous); patients who had or who eventually developed pN1 tumors had, on average, no change in T. Although the numbers are small, it is suggestive $(P=.06)$ that if distant metastatic disease were present (M1), induction therapy did not affect T. Patients with squamous cell cancers were more likely than those with adenocarcinomas to have $\mathrm{T}$ decrease with induction therapy. Multivariable analysis (Appendix Table 2) confirms these observations from univariable analysis and indicates the effect of these variables on the magnitude of change in T. Thus, the greater the depth of tumor invasion (cT), the greater the downstaging of $\mathrm{T}$. If $\mathrm{pN} 1$ tumors were found, it was highly likely that there was no $\mathrm{T}$ downstaging. With less certainty, the model predicted that $\mathrm{T}$ was downstaged in patients with squamous cell cancer more than in patients with adenocarcinoma. The analysis also suggested that linkage among the above factors accounted for the downstaging of $\mathrm{cN} 1$ to $\mathrm{pN} 0(P=.5$ for any additional information). 
Appendix TABLE 2. Multivariable correlates of the magnitude of change in $\mathrm{T}$ with induction therapy

\begin{tabular}{lrcc}
\hline Variable & Estimate \pm SE* & Odds ratio & $\boldsymbol{P}$ value \\
\hline Higher cT & $-1.8 \pm 0.41$ & $0.16 \dagger$ & $<.001$ \\
cN1/pN0 & $-0.32 \pm 0.45$ & 0.73 & .5 \\
SQ cell type & $-0.1 \pm 0.41$ & 0.45 & .05 \\
cN1/pN1 & $2.2 \pm 0.53$ & 8.8 & $<.001$ \\
cN0/pN1 & $1.9 \pm 0.72$ & 6.9 & .007 \\
Intercept 1 & $0.98 \pm 1.11$ & - & .4 \\
Intercept 2 & $4.7 \pm 1.20$ & - & $<.001$ \\
Intercept 3 & $5.6 \pm 1.22$ & - & $<.001$ \\
Intercept 4 & $6.7 \pm 1.26$ & - & $<.001$ \\
\hline
\end{tabular}

SQ, Squamous.

${ }^{*} \mathrm{~A}$ negative sign on the coefficient and an odds ratio of less than 1 indicates that the factor is associated with $\mathrm{pT}<\mathrm{cT}$.

tFor each level by which $\mathrm{pT}<\mathrm{cT}$.

\section{Discussion}

Dr Joshua R. Sonett (Baltimore, $M d$ ). Dr Rice and colleagues have in the past contributed significantly with regard to the staging, treatment, and prognosis of esophageal carcinoma. This present work continues to review their large patient population at The Cleveland Clinic with critical analysis of the results of surgical and multimodality treatment of esophageal cancer, as stratified on the basis of pretreatment clinical staging. This continued emphasis on the importance of pretreatment staging and resultant stage-specific treatment paradigms cannot be underestimated. This article, as has the group's previous work, further establishes the clinical importance and utility of EUS, and pretreatment EUS might now be considered the standard of care in the treatment of all patients with esophageal cancer.

To a mere mortal physician, the statistics are, to say the least, daunting in the article, and I will trust Dr Rice's and Dr Blackstone's analysis of the variables. However, I do have several questions regarding the conclusions that were drawn from this analysis.

In the article a final conclusion is that patients with clinically staged T1 or T2 N0 esophageal carcinoma might not benefit from induction therapy and, in fact, might be harmed. However, as you noted, the accuracy of staging T2 in the article was only $81 \%$, with a positive predictive value of only $23 \%$. The analysis of all these patients was also based on clinical staging rather than pathologic staging of the lymph nodes, a method that might additionally misstage $20 \%$ of the patients. Thus, do you think that this potential $20 \%$ error rate in accurately defining malignant lymph nodes and the inaccuracy of defining true T2 carcinomas weaken your conclusions? Could your conclusions and analysis be enhanced if the data were based on true pathologic staging, as might be gained first by fine-needle EUS analysis and then selective use of thoracoscopy or laparoscopy pa- tients within clinically staged NO disease? Can such a conclusion really be drawn from a cohort of only 11 patients?

Another interesting correlation that you have identified was the poor prognosis of those patients who continued to have N1 carcinoma after induction therapy and those patients who continued to have T3 carcinoma, N1 carcinoma, or both, either by progression of disease or lack of response. The poor prognosis of patients with residual malignant lymph nodes after induction therapy has also been seen with non-small cell lung cancer after induction therapy. Given these results, would you consider deferring surgical resection in those patients undergoing induction therapy in whom you can prove either a lack of $\mathrm{T}$ downstaging or persistence of malignant lymph nodes?

Finally, clearly $\mathrm{T}$ and $\mathrm{N}$ staging are related. As in most solid tumors, as you have shown, an increasing $\mathrm{T}$ stage will result in a higher percentage of patients with positive lymph nodes. However, despite this linkage, the correlation is not linear, and significant variability in the biologic activity and characteristics of tumors will result in earlier and/or later lymphatic or systemic failure, or both. Ultimately, molecular and biologic differences in tumor disease must play a major role in the overall prognosis and TNM stage of any tumor. Do you envision a staging system in the future that includes a biologic modification for molecular and biologic parameters?

Dr Rice. Concerning why we did not use true pathologic stage, we have to make decisions based on clinical stage. There is no doubt that there is an improvement in stratification if $\mathrm{pT}$ is used.

Fine-needle aspiration is now the standard of care. If there is an EUS-identified large node, the patient should undergo fine-needle aspiration. However, this paper discusses depth of tumor invasion, and in the analysis EUS is very good at identifying invasion beyond the esophageal wall. Whenever we use EUS, we are looking for T3 and N1. EUS and EUS/fine-needle aspiration are excellent at this identification.

As to the application of thoracoscopy, I think thoracoscopy and laparoscopy should be reserved for those patients in whom clinical stage is not obtainable by EUS or is in doubt. That is very few patients. Therefore, I would suggest that we stage all our patients with EUS and EUS/fine-needle aspiration and reserve invasive staging for those patients who have particular clinical problems.

The question about whether we know that our patients have not responded to induction therapy (ie, they have persistent N1 or T3 disease) is interesting. I think it is something we now have to address: What are we going to do with our patients who have induction therapy and persistent disease? Indeed, those patients probably should not proceed to resection.

I think that the staging system is a great advance that has allowed us to understand this disease, but it is imperfect. As we know, staging is an ongoing process. It is constantly evolving, and there is a definite possibility that molecular substaging will be added to it. 